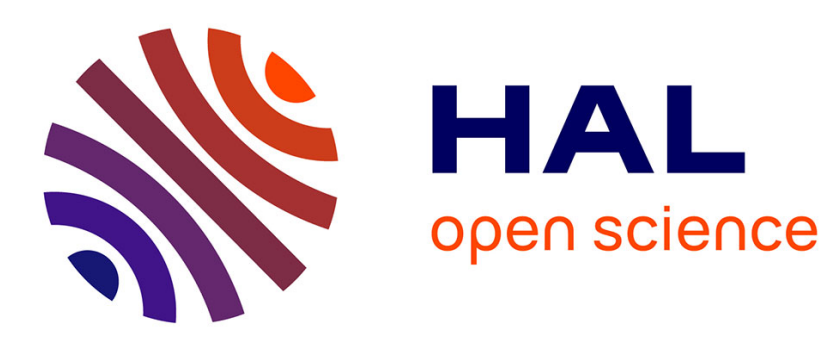

\title{
La mesure des bâtiments en Poitou à la fin du Moyen Age: première approche
}

Alain Champagne

\section{To cite this version:}

Alain Champagne. La mesure des bâtiments en Poitou à la fin du Moyen Age: première approche. Histoire \& Mesure, 2001, Mesurer les bâtiments anciens, 16 (3/4), pp.261-282. 10.4000/histoiremesure.127 . hal-01787765

\section{HAL Id: hal-01787765 \\ https://hal.science/hal-01787765}

Submitted on 19 Jun 2019

HAL is a multi-disciplinary open access archive for the deposit and dissemination of scientific research documents, whether they are published or not. The documents may come from teaching and research institutions in France or abroad, or from public or private research centers.
L'archive ouverte pluridisciplinaire HAL, est destinée au dépôt et à la diffusion de documents scientifiques de niveau recherche, publiés ou non, émanant des établissements d'enseignement et de recherche français ou étrangers, des laboratoires publics ou privés. 


\section{La mesure des bâtiments en Poitou à la fin du Moyen Age : première approche Alain Champagne}

En 1411, les échevins de Poitiers décident d'effectuer différents travaux de maçonnerie aux murailles de la ville et notamment au portail de Tison. Le prix fait passé avec cinq maçons mentionne de nombreux détails techniques ainsi que le prix de la maçonnerie construite : la toise de la maçonnerie dessusdicte, toute vees et espoisses de murs comptées, de l'espoisse dessudicte de cinq piez et demi et de six piez de long et autres espoisses de mur comptées à la toise faitte et fourni comme dessus, excepté de chaux, dont la ville fera fournir au pris de chascune toise de L s. $t .{ }^{1}$. Une lecture attentive nous apprend toutefois qu'il ne s'agit pas d'un ouvrage simple : le tarif recouvre murailles, portails et tours, avec ou sans créneaux et canonnières. Les pans de murs à édifier sont de taille et de conception différentes. Il y a donc derrière l'apparente simplicité d'un calcul de tarif à la brasse bien des questions soulevées auxquelles ce texte ne répond guère. Comment le coût d'une construction peut-il être ramené à un tarif unique, fondé sur une simple unité de longueur, défiant toute logique arithmétique ? Pourquoi ce mode de calcul est-il choisi et demeure le seul existant?

En dépit d'une bibliographie assez importante sur la construction médiévale et les métiers du bâtiment, la question du mesurage n'a pas fait l'objet d'une attention particulière. La description de la taille d'un édifice est pourtant un indice important de la manière. L'intérêt archéologique de la compréhension des techniques de prise de mesure est évident, que le bâtiment concerné soit conservé ou non. L'impact sur l'histoire des métiers du bâtiment peut lui aussi s'avérer riche, même si ce genre d'étude n'est guère développé pour l'instant.

Un usage commun aux commanditaires, aux comptables et aux maçons, témoigne d'une même approche de l'architecture et de pratiques économiques similaires, ce que montre la banalisation des méthodes de calcul. Malheureusement, les détails du mode opératoire sont alors passés sous silence dans les textes à vocation professionnelle, compliquant d'autant la tâche de l'historien. Cet article est avant tout une état de la question en Poitou. Certes, quelques points recoupent notre travail de thèse ${ }^{2}$, mais aucun dépouillement exhaustif n'a pu être mené dans les archives locales.

Les principales informations sur la prise de mesure sont issues d'un type particulier de documents, les prix faits. Ces contrats, souvent détaillés, passés entre un ou plusieurs artisans et leurs clients, fixent les conditions générales du marché. Par exemple, en 1561, Jacques Miget, marchand de Saint-Maixent, passe marché avec Georges Taunac, laboureur, pour tirer d'une pièce de terre les pierres nécessaires à la construction de 30 brasses de murs en pierres sèches, à raison de

\footnotetext{
1. Bibliothèque municipale de Poitiers (BMP), Archives de la commune de Poitiers (AP), casier 35, K4, fol. 5-6 (1411). Les unités monétaires seront ainsi abrégées : 1 . t. pour livre tournois, s. t. pour sol tournois et d. t. pour denier tournois. 2- A. Champagne, L'artisanat rural en Haut-Poitou (milieu XIVe-fin XVIe s.), thèse de 3e cycle de l'Université de Poitiers, 2000, 2 vol., 667 p.
} 
5 s. t. par brasse ${ }^{3}$. Les prix faits sont, la plupart du temps, conservés dans les minutes notariales et les archives urbaines. La documentation de la ville de Poitiers est ainsi abondante dès la fin du XIVe siècle et sera fréquemment employée. Si les fonds notariés antérieurs au second tiers du XVIe siècle sont quasi inexistants, ceux du XVIe siècle, mieux conservés, sont essentiellement urbains. Les prix faits ne sont pas les seuls documents à fournir des données intéressantes. La livraison des travaux ou le paiement des échéances prévues dans le contrat entraînent la rédaction de quittances et d'expertises, conservées notamment dans les fonds urbains et les comptes.

Le 4 octobre 1510, frère Jean de Moisy, maître d'œuvre, rend compte de l'état des bâtiments de l'abbaye Saint-Cyprien de Poitiers. Voici un extrait significatif : lequel réfectouer a quarante et troys pas ou environ de longueur et douze de largeur et sont les murailles de tous coustés avec la couverture et cherpenterie en compectante réparacion ${ }^{4} .$. Les procès-verbaux de visite, autre forme d'expertise, permettent aussi d'appréhender la manière de décrire la dimension des bâtiments parcourus. Toutes les pièces en relation avec les paiements sont riches d'enseignements. Les comptes peuvent faire mention de la mise aux enchères descendantes, de la rémunération des artisans, de la visite du maître des œuvres qui toise les travaux réceptionnés et même des prix faits ${ }^{5}$.

Les registres des délibérations municipales conservent les mandements de ces diverses opérations, où sont parfois mentionnées les prises de mesures et d'éventuelles modifications. Par exemple en 1448, une commande parmi d'autres concernant l'Université de Poitiers : commandé mandement pour la vix de la maison de l'escolle de l'eschevinage de LXX livres tournois à prandre les fondements de XII piez de par font et le seurplus qui se trouvera plus bas esd. fondemens sera paié à l'esquipolent et a esté fait led. marché en plain mois avec Gilet Aubert et Jehannin Moterin

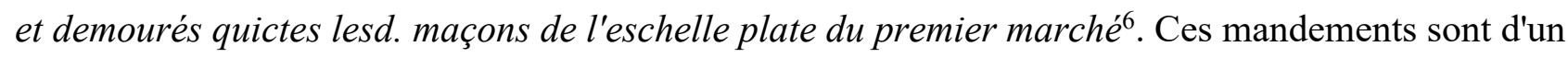
grand intérêt pour notre sujet.

A l'exposé de la documentation locale assez diversifiée, il apparaît clairement que la prise de mesures est intimement liée à des hommes de métier. Il convient donc de s'arrêter sur les moyens avant de nous intéresser aux manières de mesurer.

\section{I) Mesurer en Poitou : les outils, les hommes et les moyens}

Mesurer n'est pas affaire de novice ; aussi est-il essentiel, dès à présent, de faire le point sur les gens compétents. Leur travail, donc leurs outils, varient en fonction de la simplicité ou de la complexité de l'objet toisé. Notre attention se portera sur les hommes et leur activité puis sur les raisons du mesurage.

\footnotetext{
3- Archives Départementales des Deux-Sèvres (AD79), E2020, fol. 273.

4- R. Crozet, Textes et documents relatifs à l'histoire des arts en Poitou, Archives historiques du Poitou, t. LIII, 1942, ${ }^{\circ}$ 765, p. 201.

5. B. Giroire, L'université de Poitiers au XVe siècle, mémoire de maitrise de l'Université de Poitiers, 1977, p. 152 ; BMP, AP, casier 35, K7, fol. 35, comptes de 1456-1462 ; casier 35, K4, fol. 4 (1411).

6- B. Giroire, op. cit., p. 41 ; BMP, AP, reg. dél. n 3, fol. 189, 28 juin 1448.
} 
La documentation poitevine éclaire peu les acteurs de ces opérations. Les maîtres des œuvres ou leurs lieutenants effectuent ce genre de tâche. Ces spécialistes ont la responsabilité de certifier la bonne exécution des travaux et doivent être capables de vérifier eux-mêmes l'exacte dimension d'un corps de bâtiment ou l'épaisseur d'un mur. Le passage du maître des œuvres de la ville de Poitiers est obligatoire pour permettre le paiement d'un artisan. En 1449, à Poitiers, Jean Barbarin fournit le certificat de la bonne exécution des maçonneries de la librairie de l'université 7 . Sa fonction est celle d'un véritable expert ; son savoir-faire est lié à sa formation, celle d'un homme de métier ${ }^{8}$. Il n'est donc guère étonnant de retrouver les maîtres artisans se chargeant eux-mêmes des mesures. Entre 1503 et 1505, pour la construction de la chapelle Notre-Dame du château de Thouars, le maître maçon Jean Chahureau est responsable des opérations ${ }^{9}$. Sur les chantiers de moindre ampleur, seulement connus par des prix faits, la chose est passée sous silence. Elle est probablement le fait d'hommes de métiers, maçons ou charpentiers, sans que cela nécessite d'autre précision; nous sommes ici dans le domaine de la pratique courante. Notre connaissance des outils est toutefois marquée par les faiblesses de la documentation et malgré l'absence d'exemples, il est possible que des mesureurs puissent intervenir en cas de contestations ultérieures.

En l'absence d'expertise, d'autres archives doivent être sollicitées pour trouver des citations d'outils: lettres de rémission, comptabilités ou délibérations communales. En janvier 1475, Guillaume Regnault, charpentier de Celles-sur-Belle, est agressé chez lui par trois inconnus désirant se faire héberger ${ }^{10}$. L'un des assaillants utilise comme arme une règle. Celle-ci, faite de bois et mesurant 5 pieds (soit 1,5 m), peut être employée pour mesurer des surfaces planes dans l'atelier ou sur des chantiers.

Les mentions de cordes ou ficelles, utilisées sur les grandes hauteurs et les surfaces courbes, sont plus explicites. En 1505, le receveur du chantier de la chapelle du château de Thouars achète 40 brasses (environ $60 \mathrm{~m}$ ) de fouet pour Jean Chahureau, maître maçon. Le terme de fouet est assez rare dans cette acception, mais la suite de l'article ne laisse subsister aucun doute puisque son usage, prendre les mesures, est précisé ${ }^{11}$. Pour ce travail, les maçons utilisent généralement des cordes ou des ficelles. Celles-ci peuvent avoir différents emplois. Au château de Parthenay, en 1442, les 15 brasses (environ $24 \mathrm{~m}$ ) de petits cordeaux commandés au cordier Jean Pyaut servent à ligner les murs $^{12}$. Ici, il est donc question de marquer et de suivre des alignements lors de la construction du

\footnotetext{
7- Je, Jehan Barbarin ... certiffie à tous qu'il appartiendra avoir veu et visité une visz naguère faicte es grandes ecolles de lad. ville... ainsi qui fus à plain appert par le marché... (B. Giroire, op. cit., p. 76 ; BMP, AP, casier 31, J1034, 8 décembre 1449).

8- R. Favreau, Les maîtres des œuvres du roi en Poitou au XVe siècle, Mélanges René Crozet, Poitiers 1966, p. 1362.

9- Archives Nationales (AN), 1AP 1416-1418.

10- L. Célier, P. Guérin, Recueil des documents concernant le Poitou contenus dans les registres de la chancellerie de France, XII (1475-1483), Archives historiques du Poitou, n XLI, 1919, p. 1 (doc. 1548).

${ }^{11}$ - AN, 1 AP 1418.

12- AN, R ${ }^{1} 188$, semaines du 2 au 28 août 1442.
} 
boulevard d'artillerie. Les compas sont eux employés, soit pour mesurer, soit pour dessiner des patrons. En 1385, ceux des maçons poitevins sont destinés à faire les trez des maçons ${ }^{13}$.

Tous ces outils de mesure représentent un faible investissement et sont accessibles à une bonne partie des hommes de métier, mais leur connaissance ne suffit pas à comprendre la démarche suivie. D'autres informations sont directement issues de la description des bâtiments eux-mêmes. Mesurer un édifice nécessite quelques précisions quant à la manière de procéder. Aujourd'hui, s'il semble évident de relever largeur, longueur et hauteur, cela n'a pas été toujours le cas. Nous reviendrons sur les procédés mais pour le moment, est-il possible de savoir où sont posées les cordes et les règles pour prendre la taille d'une maison?

Il ne semble pas y avoir de règle quant au fait d'intégrer ou non les maçonneries à la dimension d'un bâtiment. A Poitiers, en 1448, la taille des grandes écoles est ainsi présentée : lesquelles escolles auront quatre vingt seze piez de long dedans euvre et vingt cinq piez de large aussi dedans euvre ${ }^{14}$. Ici, l'intérieur des murs sert de point de référence. Toujours à Poitiers, une vingtaine d'années auparavant, les mesures sont prises sur l'extérieur des tours de la porte de la Tranchée qui sont eslégiées sur le roc et sauldront (sailliront) les deux tours dix piez au dehors des meurs neux ${ }^{15}$. Dans la construction commune, seul un texte mentionne l'endroit où sont posées les cordes. L'extérieur est retenu en 1560 pour le corps de logis de la métairie de la Cupelle qui mesure 42 pieds de long et 22 de large, d'arête en arête, et 16 pieds de hauteur ${ }^{16}$. L'unique texte sur des tours et autres constructions rondes est un prix fait très tardif. En 1630, René Guillemeau demande au maçon Zacharie Ramard de lui édifier un portail en arrevoussure de Marseille et, de chaque côté du portail, une tour de 12 pieds de creux ou largeur en rond et œuvre pour un prix de 310 1. t. ${ }^{17}$. L'interprétation n'est pas très aisée, mais il est vraisemblable que la "largeur en rond" soit le diamètre de la tour et que le qualificatif "de creux" correspond au toisage effectué à l'intérieur des murs. Il n'est pas étonnant de trouver si peu de précisions techniques car ces opérations n'ont pas lieu d'être sur des documents comptables ou notariés ; seul le résultat intéresse les rédacteurs.

Prendre une mesure représente parfois un travail spécialisé nécessitant des hommes compétents et des outils adaptés. Il n'est donc pas gratuit. La plupart des mentions étant issues de prix faits, d'expertises ou de comptes; le lien avec le paiement des travaux est évident. Voici, parmi d'autres exemples, un extrait du compte de Jean Cacaud, receveur de l'abbaye Sainte-Croix de Poitiers, mentionnant le paiement de Colas de Saint-Louans, maître maçon du chantier de l'hôtel des abbesses à Vasles : A Collas de Saint Louhant et à Jehan Lambert son gendre, massons, pour avoir fait les deux pans de muraille assavoir est despuis l'église jusques à la maison de madame, excepté le petit meur ou feront les créneaux lequel est encores à fère et despuis lad. maison jusques à la

\footnotetext{
13- AN, KK 256, fol. 54, 30 janvier 1384 (1385 n. s.).

14_ R. Crozet, op. cit., p. 130, n 516 ; BMP, AP, casier 30, J973, 13 mars 1448.

15- R. Crozet, op. cit., p. 106-107, n433, BMP, AP, casier 30, J792, 8 mars 1427

16. AD79, E 2017, 1560, fol. 71.

17- AD79, E 2098, fol. 67.
} 
moitie de la chappelle de sainte Marthe ou environ. Lesquelx deux pans de mur se montent L et sept brasses de muraille sans led. petit mur, lesquelx pens ilz avoient à tasche à VII s. VI d. la brasse valant XXI l. VII s. VI d. pour ce a poyé led. receveur ausd. massons XXII l. VII s. VI d. tournois ${ }^{18}$. Dans ce cas, la mesure sert à évaluer directement le montant du marché.

Les expertises constituent souvent un moyen de rectifier une évaluation des travaux devenue caduque avec le temps. Pour l'acquittement d'un terme du contrat, il n'est alors pas rare de mentionner les différences avec le premier marché négocié et le réajustement : A Jenin Matherin et Jehan Georget, masson pour la somme de neuf cent huit livres tournois... pour ce qu'il fut neccessaire prandre les fondemens de ladicte librairie plus par font qu'il ne devoint estre prins par led. premier marché, fait led. premier marché trente et cincq toyses de fondemens, valant chacune toyse XL s. t... eslargir ladicte libraire d'un pié plus qu'il n'avoit esté divisé par led. premier marché, lesquelles avoient esté visitées et toysées par Leon Delavau, maistres des euvres de lad. ville $^{19}$. Toutefois, dès la rédaction du marché, la difficulté d'évaluer avec exactitude l'importance de l'entreprise est parfois pressentie. Des précautions sont alors prises, sous forme d'un assouplissement des conditions. En 1429, lors de la reconstruction de la tour de Tranchepié à Poitiers, les échevins imposent des fondations de 2 toises. Il est aussi prévu que l'incertitude de l'appréciation pourra être répercutée sur le prix : seront les fondemens de II toises de profont et si lesd. preneurs trouvent les fondemens plus tost ils en seront quitent et se plus bas fault aller ce sera aux despens de la ville ${ }^{20} \ldots$ Ces dispositifs concernent surtout les ouvrages qui demandent des creusements de fondations ou l'installation de puits. Pour ces derniers, la profondeur n'est qu'estimée, probablement par le sourcier. Aussi, en 1517, un contrat prévoit une modification du prix si le maçon trouve l'eau avant la profondeur convenue ${ }^{21}$. On imagine aisément que chaque partie aura à cœur de toiser le puits achevé.

Mesurer ou toiser a pour but de fixer le coût d'une construction en la décrivant dans le détail ; les mentions sont donc essentiellement conservées dans les actes de la profession. Cet acte est alors directement en relation avec une réalité économique, ce qui n'est pas sans conséquence sur les manières de prendre la mesure.

\section{II) Appréhender la mesure des bâtiments}

Dans la documentation à notre disposition, nombreuses sont les façons de toiser. Cette diversité n'est pas toujours évidente à discerner dans les textes à notre disposition, mais par recoupement il est possible de suivre l'enchaînement des opérations. Bien des questions se posent alors, portant autant sur le mode opératoire de chaque méthode de mesurage que la raison de leur usage. Elles

\footnotetext{
18- Archives Départementales de la Vienne (AD86), 2H1 45, année 1470.

19- BMP, AP, casier 35, K7, fol. 35, cmptes de 1456-1462.

20_ R. Crozet, op. cit., p. 111-12, n 448 ; BMP, AP, casier 30, J904, 31 décembre 1429.

21- AD79, E 1974, fol. 148.
} 
varient en fonction de plusieurs facteurs, notamment suivant l'édifice en question, les matériaux employés ainsi que les choix techniques induits. En conservant en mémoire ces différents points, nous aborderons donc successivement le cas des unités de longueur traditionnelles, puis des repères physiques et des modèles et enfin des modules.

\section{A) L'utilisation d'unités de longueur}

En Poitou, les unités de longueur rencontrées dans le secteur du bâtiment sont la toise, la brasse, le pied, le doigt et le pouce. Parmi celles-ci, les plus fréquemment utilisées sont la toise (environ $1,94 \mathrm{~m}$ ), la brasse (environ 1,6 m) et le pied (environ $0,3 \mathrm{~m})^{22}$. Le pied apparait beaucoup plus ponctuellement, notamment pour mesurer l'épaisseur de certains blocs de pierre ou de pièces de charpente ou de menuiserie ${ }^{23}$. Le pouce a été rencontré une fois pour affiner la mesure d'une fenêtre dans un texte de la seconde moitié du XVIe siècle ${ }^{24}$. Toutefois, les lacunes de la documentation notariale du XVe siècle nous interdisent de limiter l'emploi du pouce au seul XVIe siècle.

La manière la plus simple de décrire avec précision les dimensions d'un bâtiment est d'utiliser une de ces unités communes. Après avoir présenté les différentes possibilités de mesures d'édifices par des toisages linéaires, puis à la surface, les formes plus complexes retiendront notre attention.

\section{Le toisage linéaire}

La simple prise en compte de la longueur d'un édifice ou l'utilisation d'une toise linéaire est fréquente, mais semble limitée à quelques types de construction bien spécifiques. Pour l'édification d'un volume simple, tel un mur, plusieurs possibilités d'appréhender sa taille sont envisageables. Quand l'abbesse de Fontevrault Renée de Bourbon présente les améliorations apportées aux bâtiments du couvent entre 1491 et 1533, elle mentionne les 650 toises de mur de clôture installées $^{25}$. Ce dernier est présenté par sa seule longueur, mais il ne s'agit pas d'un document d'ordre professionnel. En 1559, Jean Rodier s'engage à construire un mur de pierre sèche pour clore la vigne de Vaize Chevalier, greffier à Saint-Maixent ${ }^{26}$. La muraille sera longue de 35 brasses, haute d'une brasse et épaisse de 2 pieds pour un prix de $6 \mathrm{~s}$. t. la brasse. Cette mesure, linéaire dans sa conception, recoupe en fait dans cet exemple une acception proche d'un volume. En effet, chaque

\footnotetext{
22. AD86, F100, imprimé du XVIIIe siècle.

23. AD86, G 982, 08 juillet 1469 ; Bibliothèque Nationale, ms. fr. 8819, fol. 63 vo, année 1432.

24- AD79, E 2025, année 1564, fol. 176.

25- R. Crozet, op. cit., p. 182, n 704 ; P. Le Nicquet, Histoire de l'ordre de Fontevrault, Paris, 1642, p. 490.

26. AD79, E 2013, fol 78. Il est possible, dans des marchés plus détaillés, de percevoir la distinction entre les fondations et la partie visible qui n'est donc plus seule à faire l'objet d'un toisage. Les réparations demandées, en 1571, à Pierre Fouasseau pour le château de Chizé sont ainsi décrites : les quatre murailles à faire sur la douve du château auront de haulteur de doze pieds hors de terre, sans comprandre es la dite haulteur les fondemans qu'il conviendra pour les dites murailles (Réparations ou travaux dans divers châteaux du Poitou, Archives historiques du Poitou, t. XX, 1889, p. 29899, 25 juillet 1571).
} 
"brasse" mesurée est un volume d'une brasse de long et de haut sur 2 pieds de large. La vocation de la "brasse" est clairement comptable car elle n'a d'autre but que de servir de base de calcul au prix.

Le toisage linéaire se retrouve aussi dans la construction des caves. En 1557, le marché de celle du logis de Jean Giraud à Saint-Maixent passé avec deux laboureurs de Saivres est très explicite ${ }^{27}$. Elle mesure 8 brasses de longueur et 8 pieds de largeur et de hauteur. Le client demande le percement d'un houys pour donner de la lumière et il fournira aux pionniers un pic à rendre en l'état pour 24 l. t. Heureusement, le notaire précise le tarif de $60 \mathrm{~s}$. t. la brasse. Grâce aux détails du contrat, il est possible de comprendre la réalité de cette "brasse" théorique et comptable. Le prix global représente l'addition de 8 brasses à $60 \mathrm{~s}$. t., prouvant que nous sommes en présence d'une mesure linéaire qui fait totalement fi des autres dimensions (largeur et hauteur). De plus, l'évaluation des aménagements induit par une cave (soupirail, escalier, porte, piédroits...) ne sont apparemment pas pris en compte. Il ne s'agit pourtant pas d'un simple cube, mais d'un édifice complexe dont on a délibérément décidé de simplifier la structure pour lui appliquer un barème moyen indexé sur la longueur. Il est obligatoirement tenu compte, à un moment donné, du tarif plus élevé des parties compliquées de la cave, mais nous ignorons quand et de quelle manière. L'usage d'une brasse linéaire et théorique est donc de simplifier le toisage.

En 1517, le prêtre Jean Augier passe, à Saint-Maixent, un marché avec Jean Dury, maçon, pour la façon d'un puits ${ }^{28}$. Celui-ci sera de 12,5 brasses $(20 \mathrm{~m})$ de profondeur, de bonne largeur et vuydance, pour une somme fixée en fonction de sa taille. Le mode de calcul de son coût est identique à celui des caves. Il est de $30 \mathrm{~s}$. t. à son entrée, et de $30 \mathrm{~s}$. t. de 3 brasses en 3 brasses, jusqu'au total de 81 . t. Ce barème donne un prix de 7 livres $15 \mathrm{~s}$. t. pour les 12,5 brasses ; les $5 \mathrm{~s}$. t. de différence ne sont pas justifiés. Nous sommes confrontés de nouveau à une brasse linéaire, indexée cette fois sur la profondeur. Le barème unique masque d'éventuelles modifications de tarif en fonction de la profondeur. Cette pratique est essentiellement mentionnée au XVIe siècle, mais cela résulte peutêtre de la conservation de la documentation poitevine.

\section{$\underline{\text { L'appréhension des surfaces }}$}

Le toisage des bâtiments offre une autre modalité technique: le calcul d'une surface et l'application d'un coefficient multiplicateur. Les structures planes sont donc particulièrement concernées.

En 1411-1412, Jehan de Rezboiseau pave plusieurs tronçons de rues poitevines toisés avec précision. Voici les termes d'un des marchés : premièrement, le pavement de la chaussé et chemin en venant du chastel de Poictiers en lad. ville, depuis le pont dorment jusques à l'enclave descendent au gué de l'eau qui vient de la Chalestre, contenant III toyses et demie de long et une toyse et demie de large, qui sont avaluées à VI toyses quarrées et troys quars de pavemens, qui

\footnotetext{
27- AD79, E 2004, fol. 194.

28- AD79, E 1974, fol. 148.
} 
vallent au pris de diz solz pour chacune toise, valant LXVII solsz VI deniers ${ }^{29}$. Les maîtres des œuvres toisent donc longueur et largeur, exprimant les mesures en toises carrées, à multiplier par un prix à la "toise", de nature comptable cette fois. L'emploi du terme de brasse carrée ou de toise carrée n'est pas obligatoire ; on lui préfère ceux de brasse ou de toise, mais ils conservent l'idée de surface. Cela ne facilite pas la compréhension des documents.

A l'instar des rues médiévales, les rues poitevines, sont rarement de parfaits rectangles. Afin d'évaluer au plus juste la surface de voie à paver, les experts de la ville et le paveur ont recours à un subterfuge. Voici ce qui est dit pour une autre portion: depuis lad. descendue dud. fossée (de Guillaume le Mercier), jusques à l'acompaignement du pavé, fait par avant, du costé du jardin Michau le Portier, contient, de long XI toises et cinq toises et demie de large à compter par son melleu, qui sont avalué à LX toises et demie qui vallent audit pris de X solsz... XXX l.V s. ${ }^{30}{ }^{30}$ Si d'un point de vue purement arithmétique, multiplier la largeur par la longueur donne une surface juste pour un rectangle, les choses sont plus délicates à gérer pour des formes non géométriques. La solution employée est de ramener le parallélépipède irrégulier à un rectangle en estimant les longueur et largeur moyennes, soit en les mesurant en leur milieu. Le souci de simplifier les démarches est bien marqué, afin d'éviter au maximum des calculs et des comptes qui pourraient devenir trop complexes. Ce qui est utilisé ici pour un pavage pourrait être aussi applicable pour des corps de maison ou des pièces d'habitation, même si leur forme est plus souvent régulière que celles des artères urbaines.

Il existe alors deux possibilités assez proches de présenter la mesure. La première consiste simplement à noter les dimensions de la maison. En 1537, par le prix fait qu'il signe, Jean Dupuys, charpentier de Pamproux, s'engage à construire une maison de 20 pieds de longueur sur 16 de largeur pour la somme de 20 écus sols ${ }^{31}$. La seconde manière appréhende quant à elle le bâtiment par son organisation et sa surface intérieure. La maison bâtie par le laboureur Mathurin Pinaudeau comporte six chambres sur deux niveaux : trois de 18 pieds au carré et les autres de 13 pieds de largeur sur 18 de longueur en œuvre ${ }^{32}$. Cette méthode est autant applicable à des surfaces au sol qu'à des surfaces projetées sur des murs ; cependant, dans ce cas, la documentation est moins riche. En effet, les contrats proposent des prix globaux, empêchant l'interprétation du mode de calcul, et lorsque par chance les prix sont détaillés, il nous manque les mesures du bâtiment. A Vasles, sur le chantier de l'enceinte de l'hôtel des abbesses de Sainte-Croix de Poitiers, le receveur paye premèrement pour la main et tache de Collas de Saint Louent, masson, lequel avoit prins à tashe à fère lad. besoigne sans despens au pris de XI s. VIII d. la basse, et doit led. masson fournir de toute taille laquelle muraille avecque lad. portau et tonelles se montet IIII XX XVI brasses et demye de

\footnotetext{
29- A. Luceau, Compte poitevin de 1411-1412, mémoire de maîtrise de l'université de Poitiers, 1973, p. 25 ; BMP, AP, casier 35, K4, fol. 10 .

30- A. Luceau, op . cit., p. 25 ; BMP, AP, casier 35, K4, fol. 10.

31- AD79, E 1975, fol. 285.

32- AD79, E 2040, 1570, fol. 261.
} 
muraille valant aud. pris de XI s. VIII d. chacune brasse LVI l. IIII s. II $\mathrm{d}^{33}$. Il peut s'agir de brasses linéaires comme de brasses carrées. Son prix est une moyenne qui globalise différents tarifs, car les travaux recouvrent une grande variété de murs de diverses épaisseurs, un portail et des tours.

S'il n'est guère envisageable, aujourd'hui, de chercher à comprendre comment les maîtres d'œuvre parviennent à globaliser les prix, il est possible en revanche d'appréhender la manière dont la taille de bâtiments à la structure complexe sont présentées.

\section{Le toisage des maçonneries complexes}

Bien que les voûtes sur croisées d'ogives soient couramment rapportées ${ }^{34}$, leurs dimension et description sont rarement précisées. Seul le contrat signé, en 1599, par les frères Orgeon, maçons, en vue d'améliorations à porter dans la maison d'Hélie Martin, signale la construction d'une voûte de 15 pieds de creux et de 60 pieds de longueur ${ }^{35}$. Deux mesures apparaissent donc : d'une part la longueur de la voûte, peut-être en projection au sol, ce qui correspondrait alors à la longueur de la salle, et d'autre part, la hauteur de la voûte, soit de la clef au sol, soit de la clef aux reins de la voûte. Ces deux données paraissent suffisantes aux maçons pour permettre l'édification de cette partie de la construction.

De longues descriptions techniques complètent alors les contrats, comme pour n'importe quel édifice. Les fondations devront atteindre le $\operatorname{rocher}^{36}$ ou une profondeur fixée à l'avance ${ }^{37}$. Chaque ouverture est également toisée : la porte d'un mur au portail de Tizon devra avoir huit piez, vostée dedans et dehors de onze pies de hault ou environ ${ }^{38}$. La taille des corbeaux soutenant l'étage une tour est de même précisée, 3,5 pieds de long sur 1,5 pieds de long et 3 poiz de large. Des modalités de mesure identiques à celles de maçonneries simples sont alors appliquées aux fenêtres, portes et escaliers (taille et nombre des marches) ${ }^{39} \ldots$

Il est possible d'associer deux méthodes de calcul de prix, et donc de toisage. En 1411, le prix fait des réparations des murailles de Poitiers, à la porte de la Tranchée, sont décrites sans aucun toisement. Le contrat stipule ensuite que les preneurs devront fournir toutes les fournitures et que parmi ce que la saillie des troys taux d'encorbellement jusques à la hauteur du dessoubz des couvertures et bées, ne se comptera point la toise, et aussi les parpains du mur qui seront faiz entre le groz mur et les couvertures jusques à l'arazement du gros mur; et sera compté en maçonnerie ce qui se escorchera (percera) à l'encontre dud. pan de mur, les troys toises pour une et chacune toise

\footnotetext{
33- AD86, 2H1 45, comptes de Jean Cacaud, année 1471.

34- AD79, E 1994, marché Prévot-Bonnet; id., E 2002, fol. 221 ; id., E 2025, fol. 176.

35- Soit environ 4,5 m sur $18 \mathrm{~m}$ (AD79, E 2072, fol. 593).

36. et sera tenu ledit preneur de faire toute délivrée des nouveaux fondemens jusques à la tine et rochier (A. Luceau, op. cit., p. 15-17 ; BMP, AP, casier 35, K4, fol. 5). Une grange de 20 pieds de largeur et de 32 pieds de longueur en œuvre, en creusant à leurs dépens des fondations jusqu'à la tine (AD79, E 2039, fol. 323).

37- Cf. note 20.

38 - A. Luceau, op. cit., p. 15-16 ; BMP, AP, casier 35, K4, fol. 5 vº.

39 - Eschailles qui montera jusques es entrees des haultes escolles ou il y aura XXIX à XXX marches et aura chacune marche cinq piez dedans euvre... (B. Giroire, op. cit., p. 76 ; BMP, AP, casier 31, J1034, 8 décembre 1449).
} 
au prix de 4 l. 10 s. $t .{ }^{40}$. Le prix à la toise indique donc que tous les travaux sont ramenés à un étalon commun. Des coefficients multiplicateurs sont probablement appliqués aux différentes maçonneries, mais un seul est connu. Le même prix sera appliqué pour la destruction de trois toises d'anciens créneaux et pans de murs ou la construction d'une toise. Ce barème prend en compte la particularité de travail qu'induisent ces deux opérations. Il reste rare que la documentation poitevine fournisse les détails du mode de calcul du prix final, même si cette façon de calculer est bien attestée.

L'usage d'unités de mesure communes, largement répandu dans le Poitou médiéval, demande, bien entendu, à ce que ces unités aient une valeur reconnue et fixe, vaste question mais hors de ce propos. La description précise de maçonneries complexes nécessite probablement des connaissances accrues. Peut-être est-ce la raison pour laquelle d'autres méthodes émergent aussi plus ponctuellement, notamment l'utilisation de repères matériels caractérisés par l'usage.

\section{B) Les repères matériels}

L'usage de tels repères, d'exemples ou d'objets connus de tous, apparaît parfois dans les prix faits de plusieurs types d'édifices. Il s'agit, pour les parties en cause, d'être aussi précis que possible tout en excluant le passage à un toisage. Il peut y avoir dans cette pratique le signe d'un recours au bon sens commun qui simplifie toutes les procédures et les vérifications au moment de la livraison de la commande.

A Angoulême, deux pierriers s'engagent en décembre 1540 à creuser la cave de Denis Delage. Elle sera dotée d'un escalier et d'une porte de la largeur de entrer une pippe de vingt à laise ${ }^{41}$. L'Angoumois est alors un pays de vin, au même titre qu'une bonne partie du Poitou, et la taille d'une pipe de vin est un excellent repère laissant peu de place à d'éventuelles contestations. L'homme ou l'animal servent aussi de référence. En 1525, une description de la ville de Vouvant détaille le portail le portail du château : un pont levys et une porte pour entrer une beste chargée et ung homme dessus à cheval ${ }^{42}$. La notion de confort du passant est aussi prise en compte puisque certains portillons doivent être fait pour laisser passer un cavalier sans baisser la tête ${ }^{43}$. La taille d'un individu sert aussi à déterminer la hauteur du mur intérieur d'une chambre de l'université de Poitiers : faire un mur de stablement de la haulteur d'un homme ou environ ${ }^{44}$. L'économie et la recherche de la simplicité semblent à l'origine de l'usage de modèles dans la construction.

\footnotetext{
40- A. Luceau, op. cit., p. 10 ; BMP, AP, casier 35, K4, fol. 4 v.

41- Archives départementales de la Charente (AD16), $2^{\mathrm{E}}$ 965, 26 décembre 1540.

42. AD86, C548, fol. $5 \mathrm{v}^{\circ}$.

43- AD79, E 2098, fol. 67, 1630-32.

44- F. Gaulon, Les comptes extraordinaires de la ville de Poitiers (1456-1462), mémoire de maîtrise de l'université de Poitiers, 1977, p. 253-55. BMP, AP, reg. dél. n 4 fol. 277, 22 mars 1465 (n. s.).
} 
Le même but motive le renvoi à des constructions à l'identique. A Poitiers, les fortifications de la porte de Tizon seront assis les parpains et créneaux, par la forme et manière qu'ils sont à présent ond. viel portal ${ }^{45}$. La copie peut être demandée dans la construction courante pour une seule mesure. La cave voûtée de Jacques Biot, marchand tanneur de Saint-Maixent, sera de la même profondeur que celle de la maison mitoyenne de la sienne ${ }^{46}$. Quand la copie porte sur tout ou partie d'une construction, elle marque alors plus la notoriété d'un artisan auquel il est reconnu une marque de fabrique, plutôt que le désir d'économiser ${ }^{47}$. Ainsi, en 1552, Pierre Bonnet, maçon, construit à la maison de Pierre Prévost, une "croisée et demie" semblable à celle qu'il a déjà faite chez Simon Valéry et une cheminée identique à celle qu'il a bâtie au logis de Pierre Courtineau ${ }^{48}$.

L'usage de repères physiques et de la copie sont plus spécifiquement associés à des œuvres de maçonnerie. Les travaux de charpenterie n'ont pas, jusqu'à présent, été mesurés et rencontrés, car d'autres systèmes lui sont appliqués.

\section{C) Le cas des modules dans la construction rurale}

L'existence de modules pour préciser la taille des maisons est délicate à cerner. En effet, l'historien doit être capable d'interpréter un vocabulaire, récurrent et formel, pour y entrevoir une réalité technique. L'approche modulaire de la mesure se rencontre dans plusieurs types de documents : quelques rares prix faits ou transactions (suite à des contestations) ${ }^{49}$, et essentiellement des contrats de reconstruction -en fait des arrentements de terre en friche, accompagnées de l'obligation de construire. Ce ne sont pas à proprement parler des marchés de construction ; en effet, l'objectif primordial est de fixer les clauses de mise en culture des parcelles et les parties en présence ne sont pas des gens de métiers. Ce caractère quelque peu informel, du point de vue de l'historien de la construction, doit être gardé en mémoire, mais il reste néanmoins utile de prendre en compte ces témoignages.

Voyons l'un de ces textes. Le 6 août 1493, à Vasles, l'abbesse de Sainte-Croix de Poitiers arrente à Jean Maréchal, seigneur de la Sayette, une terre en friche et gast ${ }^{50}$. Parmi les clauses particulières, le preneur sera tenu faire ou faire faire une maison en lad. pièce de terre de trois travées et à fays convenable pour faire demeurant dedans trois ans prochains venant. Des années 1430 jusqu'à la première décennie du XVIe siècle, ces actes sont fréquents dans les chartriers poitevins et, de

\footnotetext{
45- A. Luceau, op. cit., p. 16-17 ; BMP, AP, casier 35, K4, fol. 6.

46- AD79, E 1978, fol. 208. La tour à bâtir, en 1558, au logis de Coutaux sera de la même hauteur que la tour voisine (AD79, E2002, fol. 221)

47- Marché passé par Maixent Monnet, en 1560, pour un pressoir à vin qui est dit comme celui de la maison de Jacques Rivet (AD779, E2016, fol. 92). Marché passé en 1585 par Pierre Peing pour une maison neuve à la Morlière, avec une cheminée semblable à celle que Pierre Portraut a fait bâtir dans la même paroisse (AD79, E2062, fol. 12).

48 - AD79, E1994, sans folio.

49- AD86, $E^{3}$ 15, 14 mars 1439. Transaction suite au retard pris par un charpentier qui devait construire une charpente de cinq travées à Latillé.

50_ AD86, 2H 1 liasse 46, n 151, 6 août 1493.
} 
manière plus générale, en France ${ }^{51}$. Un délai d'un à dix ans est alloué pour la réalisation de maisons de deux à quatre travées. Les actes sont presque semblables, si l'on excepte quelques détails sur les matériaux de couverture et l'obtention de droits d'usage ${ }^{52}$.

Mais revenons maintenant aux "travées" proprement dites. Ce terme n'est pas unique. Ceux de tretz ou trez, aussi rencontrés, signifient poutre ou solive ${ }^{53}$. On a un premier indice de définition de la travée, en liaison étroite avec la charpente. Quelques baux détaillés fournissent des indications complémentaires sur leur montage. En 1460, Jean Jousserand, seigneur de Lairé, arrente à Jean Caillet les restes du hameau de Beaulieu contre diverses redevances et l'obligation de bâtir dans les 10 ans une maison de deux travées de long, fermée de murailles ${ }^{54}$. L'intérêt de ce document est de situer les travées dans l'axe longitudinal de la maison. En 1478, à Vasles, il est question d'une grange avec appentis posé sur un des côtés ${ }^{55}$. Ces appentis, très souvent mentionnés dans les contrats, augmentent donc la surface des bâtiments dans leur longueur, mais ne sont pas comptés en travées, ce qui confirme le caractère technique de cette appellation.

Si sur l'ensemble du corpus étudié, quasiment tous les actes, soit plus une centaine, rapportent la présence ces appentis, un seul document détaille les dimensions des travées. En 1438, l'abbaye du Pin impose, à Jean Gaullot, la construction d'une maison de troys trez de trente piez de long et vingt piez de large, soit environ une maison de $9 \mathrm{~m}$ sur $6 \mathrm{~m}$ et une travée d'environ $3 \mathrm{~m}$ sur $6 \mathrm{~m}^{56}$. Face au silence des autres baux, ce document étonne par sa précision. Les raisons de celle-ci demeurent énigmatiques : date du bail très précoce par rapport à la chronologie de la reconstruction poitevine, lubie de notaire, preneurs inconnus ou étrangers et ignorant les usages locaux ou bien travée d'une longueur et largeur inhabituelle? La dernière solution n'est pas solide au vu d'autres textes. Ainsi, une sentence autorisant la délivrance de bois de ligne dans la forêt de Chizé mentionne une grange

\footnotetext{
51- Les actes demandant la reconstruction d'édifices se rencontrent un peu partout : en Bordelais (R. Boutruche, La crise d'une société : seigneurs et paysans du Bordelais pendant la guerre de Cent Ans, Paris, 1947, p. 404), en Normandie (Beaurepaire (C. Robillard de), L'état des campagnes de la Haute-Normandie dans les derniers temps du Moyen Age, Evreux, 1865 (rééd. 1981), p. 302, 322, 332), en Verdunois (A. Girardot, Le droit et la terre : le Verdunois à la fin du Moyen Age, Nancy, 1992, p. 798-99), en région parisienne (G. Fourquin, Les campagnes de la région parisienne à la fin du Moyen Age du XIIIe s. au début du XVIe s., Paris, 1963, p. 447), en Quercy (J. Lartigaut, Les campagnes du Quercy après la guerre de 100 ans (vers 1450-1500), Toulouse, 1978, p. 122-23), en Auvergne (P. Charbonnier, Une autre France : la seigneurie rurale en basse Auvergne du XIVe au XVIe siècle, Clermond-Ferrand, 1980, p. 530) en Limousin (J. Tricard, Les campagnes limousines du XIVe au XVe siècle : originalité et limites d'une reconstruction rurale, Paris, 1996, p. 142, 205), mais aussi en Sologne, Bourbonnais et Saintonge (cf. note 52).

52- AD86, $\mathrm{E}^{2} 111,11$ février 1461.

53. AD86, reg. 22 sup., n 61, p. 90, 28 octobre 1438. Ce vocabulaire est aussi employé en Saintonge (J. Glénisson, La reconstruction agraire en Saintonge méridionale au lendemain de la guerre de Cent Ans, Revue de la Saintonge et de l'Aunis, t. 1, 1975, p. 75). En Sologne, il est par contre question de chez ou ferme de maison (I. Guérin, La vie rurale en Sologne aux XIVe et XVe s., Paris, 1960 p. 247). En Bourbonnais, R. Germain rencontre des maisons à plusieurs chaptz et tirans (R. Germain, Les campagnes Bourbonnaises à la fin du Moyen Age (1370-1530), Clermont-Ferrand, 1987, p. 339, 341). En Bordelais, la taille des maisons est évaluée en nombre de pièces (R. Boutruche, op. cit., p. 404). Le terme de chas ou chaps signifie maison, partie de cuisine, bâtiment léger d'après F. Godefroy (Dictionnaire de l'ancienne langue française... Paris, rééd. 1938, t. II, p. 83).

54- Le hameau est aussi appelé Challebert (AD86, E²111).

55. Une grange de deux travées garnie d'un appentis sur un des cousté dicelle (AD86, reg. 22 sup., n 49, p. 73, 22 décembre 1478).

56- Soit 10 pieds sur 20 (AD86, reg. 22 sup., n 61, p. 90, 28 octobre 1438).
} 
de 40 pieds de long, soit exactement quatre travées de 10 pieds sur 20 ( $3 \mathrm{~m}$ sur 6$)^{57}$. Il y a en outre corrélation avec l'histoire des charpentes et l'ethnologie. L'existence de ce module est liée aux techniques de montage car la travée induit une distinction, dans les murs de la maison, entre les poteaux cormiers et les supports secondaires ${ }^{58}$. Son intérêt est d'économiser les bois de forte section utilisés pour les cormiers, les arbalétriers et les entraits. Il y a deux arbalétriers et un entrait par travée, et dans les intervalles seulement des chevrons non porteurs, de petite section, reposant sur des sablières et une faitière ${ }^{59}$. La même logique conditionne l'emploi de la ferme de charpente ou du tiran (entrait) comme module en Sologne ou en Bourbonnais ${ }^{60}$. La multiplication des conflits autour des forêts dans la seconde moitié du XVe siècle peut, en partie, être lié à la volonté d'économiser du bois d'œuvre, d'important diamètre et de grande valeur monétaire, expliquant le développement d'un module de charpente. En effet, les travées à entrait limitent l'écartement des murs, et donc la largeur des bâtiments, à la portée maximale d'une pièce de chêne. Pour passer outre, il faut avoir recours à des assemblages plus complexes. Une portée de $6 \mathrm{~m}$ environ correspond tout aussi bien à la largeur des maisons paysannes poitevines et qu'à la taille des travées demandées par l'abbaye du $\operatorname{Pin}^{61}$. Cette contrainte technique rend quasiment inutile la précision de leur longueur qui, dès lors, devient commune.

Le nombre des maisons à bâtir fait penser à de véritables lotissements. Le développement de la travée, non seulement comme moyen d'appréhender la taille de l'habitat paysan, mais aussi comme technique d'assemblage, est le signe d'une certaine forme de standardisation. Ce n'est donc pas un hasard qu'elle soit passée dans les mœurs au point d'être conservée comme module de base. L'emploi du terme travée en témoigne. Des expertises d'officiers forestiers et de charpentiers, en vue de la délivrance de bois d'usage, ont été, par chance, sauvegardées. En 1475, François Maynet, charpentier, évalue le bois de ligne nécessaire à quatre travées de maisons garnies d'appentis avant que les sergents et verdiers de la forêt de Chizé fassent de même pour le bois de deux travées de

\footnotetext{
57- Je remercie M. Favreau de m'avoir communiqué ce document (R. Favreau, La forêt de Chizé au Moyen Age, Actes $d u 87^{e}$ congrès national des sociétés savantes, section histoire et philologie, 1962, 1963, Paris, p. 180, n. 1 ; AN, $\mathrm{P} 1409^{3}, \mathrm{n}^{\circ} 872,4$ avril 1474). Ce raisonnement a ses limites puisqu'en 1570, d'autres granges mesurent 32 pieds de long sur 20 de large (AD79, E2039, fol. 62).

58. J. Chapelot, R. Fossier, Le village et la maison au Moyen Age, Paris, 1980, p. 312.

59- La faîtière est rarement mentionnée. Signalons le contrat d'arrentement du 31 octobre 1470 passé entre le chapitre de Saint-Pierre le Puellier de Poitiers et Jean Blois de Saint-Germain de Poitiers, et obligeant ce dernier à faire une echauffetour (pièce avec cheminée) à faye à deux travées (AD86, G1767).

60_ En Sologne, il est question de maisons à trois fermes, correspondant à un module de charpente alors qu'en Bourbonnais, R. Germain rencontre des maisons à plusieurs tirans (I. Guérin op. cit., p. 247 ; R. Germain, op. cit., p. 339, 341). La ferme est l'assemblage des pièces de charpente dans un plan vertical portant le faîte d'un comble.

61- S. Jean, L'architecture rurale française, corpus des genres, des types et des variantes : Poitou, Pays Charentais, Paris, 1987, p. 141, 152, 171, 203. Les fouilles archéologiques d'Airvault-Soulièvres ont aussi confirmé cette largeur de maisons et de granges de la fin du XIVe siècle au début du XVIe siècle (A.-M. Fourteau-Bardaji, J.-P. Nibodeau, B. Vequaud, Aux origines d'une paroisse : les fouilles archéologiques d'Airvault-Soulièvres, Les clefs du patrimoine, $\mathrm{n}^{\circ} 3$, Parthenay, 1994, p. 28, 36-37). Cette taille maximale est aussi confirmée par les fournitures de pièces de charpentes trouvées dans les redevances ou les actes notariés. En 1469, les solives (pièces de bois portant les planchers) achetées font de 12 à 26 pieds, soit de 3,6 m à 6,3 m). En 1512, le paiement d'une dette est effectué en solives de chêne de 21 pieds (AD86, G 982, 8 juillet 1469 ; AD86, E² sup. 216, $1^{\mathrm{er}}$ mai 1512).
} 
maisons... et le boys de troys travées de maisons garnies de soliveaux de planches et de lates ${ }^{62}$. Notons d'abord le pluriel du mot maison; dans l'expression "travées de maisons", il est systématique. Il s'agit peut-être d'une erreur, mais il témoigne de l'importance de cette expression. La travée n'est plus un simple complément de nom, mais le nom autour duquel la syntaxe de la phrase s'organise. L'objet du contrat n'est plus la maison à construire, mais le nombre de travées de maison que les experts vont accorder à l'usager. Elles deviennent ici l'objet de la transaction, au même titre que, dans une comptabilité, un maçon sera payé pour la construction de 10 brasses de murs. Peu importe alors qu'il s'agisse d'un, deux ou trois murs. Le module apparaît bel et bien comme une unité et non plus comme une simple indication technique. A ce stade, opter pour un paiement à la travée n'est qu'une petite étape supplémentaire ${ }^{63}$. Le vocabulaire est partagé par les habitants qu'ils soient artisans charpentiers, mais aussi officiers seigneuriaux, notaires ou paysans. Il est donc pratique et adapté aux besoins.

L'usage des travées n'est pas l'apanage des seules maisons paysannes ; ce système de charpente est aussi employé pour la construction d'une halle de quatre travées à Champagne-Saint-Hilaire, en $1481^{64}$. En tout état de cause, ce système de mesure basé sur un module commun est essentiellement lié à la construction des charpentes, mais pas obligatoirement à des maisons entièrement en bois ${ }^{65}$.

Les manières d'exprimer la mesure sont par conséquent multiples et peuvent apparaitre conjointement sur un même chantier et dans un même contrat. Au moins trois semblent bien identifiés :

- la première est l'utilisation d'unité de mesure conventionnelle, avec un jeu complexe et assez peu compréhensible en Poitou d'évaluation du prix de l'édifice. En effet, si pour simplifier l'estimation, l'usage d'un étalon (toises linéaires ou toises carrées) est un fait acquis, la réalité des procédés nous échappe totalement.

\footnotetext{
62- Je remercie M. Favreau de m'avoir communiqué ce document. AN, P $1409^{3}, \mathrm{n}^{\circ} 872^{5}, 4$ avril et 21 décembre 1475.

63- Toutefois, il n'a jamais été trouvé de prix à la travée, peut-être du fait des prix toujours globaux et du fait de la rareté des textes mentionnant des tarifs.

64_ AD86, G 527, fol. LIII r ${ }^{\circ}$; R. Crozet, op. cit., p. 172, $\mathrm{n}^{\circ}$ 667. cum hoc tenebitur dictus Bertrandus facere et eddificare aut fieri et edificari facere infra unum annum immediate sequentem unum auditorium quator travearum laice halles dictore loci terre et domum de Campigniaco Sancti Hilarii suis propriis sumptibus et expensis.

65. Une autre méthode pour appréhender la taille d'une charpente a été rencontrée une seule fois, dans un prix fait de l'université de Poitiers. Celui-ci stipule que la charpenterie de la couverture sera faicte à sept quartiers tant en longueur que en largeur selon les murailles comme il appartient... Item sera faicte la plate forme de lad. charpenterie à blochez et à fest et soufest à VII quartiers ainsi qu'il appartient et troys tirans qui seront mis aux lieu le plus neccessaire...Item sur chaque mur aura double sablère qui portera plate forme de lad. charpenterie avecque et au plus nectessaire qui seront à sept quartier et y seront faicte deux lucarnes pour donné clarté... (B. Giroire, op. cit., p. 159 ; BMP, AP, caiser 32, J1224, 23 février 1460 (n. s.)). Il est étonnant de constater que les quartiers sont aussi nombreux en largeur qu'en longueur, pour un bâtiment pourtant de forme rectangulaire. Les nombreux emplois de ce mot, pour du bois fendu en quart, des pierres de taille ou le quart, ne permettent guère d'interpréter ce document. Au vu de la description, il s'agit d'une charpente à chevrons-formant-ferme avec des entraits (Fenêtres sur combles, catalogue d'exposition, conseil général de la Vendée, 22 juin au 31 octobre 1995, éd. de l'inventaire, 76 p.). Ce mode de montage est typique des progrès de l'assemblage des grandes charpentes de la fin du Moyen Age et du XVIe siècle, pourtant, dans l'état actuel de nos connaissances, il n'a pas été possible de trouver des parallèles aux quartiers de charpente ici mentionnés.
} 
- la seconde permet de simplifier encore les démarches. Effectivement, en prenant en considération un modèle déjà bâti, les toisages sont évités : il suffit de construire à l'identique. Toutefois notre ignorance du calcul du coût est réelle.

- la troisième voit l'utilisation d'un module standard, probablement issue de l'assemblage des charpentes et de la volonté de rationaliser la construction commune. Si les travées n'ont peut-être pas permis d'évaluer un prix de revient d'un bâtiment, elles ont probablement servi à mesurer le volume de matière première que nécessitait telle ou telle maison à bâtir.

En dépit de ses lacunes, la documentation poitevine permet de retracer un fidèle tableau des moyens de mesurer les constructions aux XVe et XVIe siècles. Toutes semblent largement admises. L'utilisation d'unités de mesures classiques est largement répandue et donne un aperçu des techniques du toisage. Ce dernier entraîne un recours fréquent à de notables imprécisions qui visent toutes à laisser la prépondérance à une simplification du calcul des prix. Elles ont été constatées pour le pavage, les caves, les puits et les travaux de l'enceinte de Poitiers. L'utilisation d'une seule unité évite d'avoir à négocier une multitude de tarifs pour les parties complexes. Des calculs, qui nous échappent, permettent par un jeu d'équivalence de tout ramener à un tarif unique dans une unité de compte. L'appréciation de la valeur de l'édifice se trouve facilité, même si elle devient plus approximative, mais dans la mesure où aucune contestation majeure ne nous est parvenue, on peut supposer que tous les intervenants y trouvaient leur compte.

Les caractéristiques de la construction en Poitou, en particulier dans le monde rural, sont à rapprocher de l'usage d'un module. Dans cette région, l'habitat rural utilise encore essentiellement le bois comme matériau de construction. L'emploi de la travée, en relation avec la charpente, semble largement dominer dans les campagnes. Quelques exemples, moins nombreux, sont aussi connus en ville. Sa présence est à mettre en rapport avec le choix du matériau et la modernisation des systèmes d'assemblages. Les documents professionnels illustrent le choix existant entre un toisage classique et le module lié à la charpente.

Chaque méthode est révélatrice d'une réalité économique. Au-delà du développement de la ferme dans la charpente réapparaît la volonté de ne pas gaspiller la matière première. De plus, il évite de faire appel à des experts en cas de conflit tant le comptage des travées est simple. L'étalon de mesure (la toise linéaire ou carrée) permet aussi de simplifier le calcul du prix des marchés, en faisant fi de toute rigueur cartésienne. Cette volonté trouve son origine dans le mouvement de standardisation de la production. Quant à l'absence de mesureurs, elle trouve peut-être un début d'explication dans la faible importance des entrepreneurs privés. En dépit de l'essor économique lié à la reconstruction et à l'attrait du secteur artisanal, l'importance des investissements des élites poitevines n'est pas exceptionnelle. Poitiers est avant tout une ville d'hommes de loi et d'administration, où l'activité économique n'est pas comparable avec celle de villes commerçantes 
comme La Rochelle, pour ne citer que sa grande voisine et rivale ${ }^{66}$. Le système des enchères descendantes — pour tous les marchés urbains — pousse à la rentabilité extrême, oblige à calculer au plus juste et favorise toutes les solutions visant à diminuer les coûts ${ }^{67}$.

Des systèmes probablement proches se rencontrent dans d'autres secteurs non explorés. Le calcul du prix des vitraux de l'université de Poitiers présente un système d'équivalence par quartier encore bien obscur ${ }^{68}$. De larges perspectives de recherche sur la question des mesures s'offrent aux chercheurs. Une exploration plus complète des archives poitevines est on ne peut plus nécessaire pour compléter et achever un inventaire exhaustif.

\footnotetext{
66- R. Favreau, La ville de Poitiers à la fin du Moyen Age - une capitale régionale, mémoire de la société des antiquaires de l'Ouest, t. XIV-XV, $4^{\mathrm{e}}$ s., 1977-78, p. 438, 523-31; A. Champagne, L'artisanat rural en Haut-Poitou (milieu XIVe-fin XVIe s.), thèse de doctorat de $3^{\mathrm{e}}$ cycle, université de Poitiers, p. 450-51, 464.

67. Cela a été remarqué à Alençon (J. Dubois, Notre-Dame d'Alençon à la fin du Moyen Age : financement et reconstruction - thèse de l'université de Haute-Bretagne, 1997, p. 368, 379.

68- Item, et aura es deux pignon qui feront les deux boutz, en chacun, en chacun d'iceulx, ung vitral de pierre de taille et en chacun vitral aura trente ung cartier, chacun de deux piez de long et d'un pié de hault, revenant à huit piez, et aussi, le moyenneau à la haulteur desditz huit piez... (BMP, AP, casier 30, J973, 13 mars 1448. Publié dans Marcel Fournier, Les statuts et les privilèges des universités françaises depuis leur fondation jusqu'en 1789, t. III, Paris, 1892, p. 308).
} 